\title{
Turbulence averaging within spark ignition engines
}

\author{
P. Sullivan, R. Ancimer, J. Wallace
}

\begin{abstract}
This paper examines velocity averaging within Spark-Ignition (SI) engines, a non-stationary system. Comparison is made between the mean and turbulence velocities found from (a) Ensemble, (b) Cyclic and (c) Wavelet-based averaging. The various methods of extracting turbulence within this flow system result in qualitatively similar average velocities; however, there are significant differences in the turbulence velocities and spectral content of the flow field based on the definition used. The differing interpretation of turbulence results in a subjectivity to the physical understanding of the flows. The experience in extracting coherent structures in stationary turbulence suggests that wavelet analysis offers a unique insight that has applicability for engine studies.
\end{abstract}

\section{1}

\section{Introduction}

SI engine flows are inherently non-stationary due to the piston and valve motions. Analysis of these flows involves separating the flow velocities into (a) large scale motions (the mean) which act to convect and to strain the flame kernel and (b) small scale motions (the turbulence) which act to deform the flame kernel, see Fig. 1. The convection, strain and deformation of the flame kernel have a strong influence on the ultimate performance and emission characteristics of SI engines (Fansler and French 1987; Hall and Bracco 1987). The flows are non-stationary in both the small and large scale motions making it difficult to decompose the effect of the mean and turbulence on the combustion process.

\section{Received: 25 February 1998/ Accepted: 12 August 1998}

P. Sullivan, R. Ancimer, J. Wallace

Engine Research and Development Laboratory

Mechanical and Industrial Engineering, University of Toronto

Toronto, Ontario M5S 3G8, Canada

Correspondence to: P. Sullivan

The authors gratefully acknowledge the financial support of British Gas and the Natural Sciences and Engineering Research Council (NSERC) of Canada. The optical access cylinder head for the L-head CFR engine was designed by Hannu Jääskeläinen and Alvin Cheung. The efforts of Dr. Mauricio Dziedzic in commisioning the LDV system are also greatly appreciated
Three different averaging techniques have been chosen for this study to decompose the flow: Ensemble and Cyclic averaging (Fansler and French 1987; Hall and Bracco 1987; Catania and Mittica 1989) and a new wavelet based averaging method (Wiktorsson et al. 1996; Sullivan et al. 1996; Söderberg and Johansson 1998). Ensemble and Cyclic averaging were chosen because they are more commonly used within the literature. Each averaging technique decomposes the flow into mean and turbulence velocities in a different manner. Thus, the choice of technique affects the interpretation of the flow in SI engines. For example, while the ensemble mean velocities are qualitatively similar for Ensemble and Cyclic averages, the turbulence velocity, corresponding turbulence energy and spectral extent of the turbulence are significantly different. These differences have implications on the numerical modelling of the flow as comparison with experimental results is not direct because of differences in flow definitions. The introduction of the wavelet decomposition technique provides an improved and localised understanding of the turbulence.

In the discussion of the three techniques used for extracting turbulence, filtering frequencies will be described in terms of (Engine Cycle) $)^{-1}$. An engine has an operating frequency, $\omega$, which indicates the crankshaft rotation in radians/second. For a four-stroke engine, the cycle frequency would be $\omega / 2$. In the experimental work performed, velocity measurements were taken every crankshaft degree (CAD) or 720 samples/ Engine Cycle. Thus, the measurement sampling frequency was $360 \omega$.

Specifying filtering frequency in terms of (Engine Cycle) $)^{-1}$ allows the use of integer values for the frequencies under consideration and focuses attention on the individual cycle rather than an absolute number that changes with engine speed. In effect the numerical value is the number of bins into which the data from an individual engine cycle has been grouped, e.g., 10 (Engine Cycle) $^{-1}$ indicates that the data has 72 CAD width.

\section{1}

\section{Computational and experimental work}

Both numerical modelling and experimental work have shown well defined structures within engines that act to control combustion (Hall and Bracco 1987). Comparisons between numerical and experimental results are difficult to make because of different flow definitions: computational flows tend to be Ensemble averaged, and experimental flows tend to be Cyclic averaged. Computational models, e.g., KIVA (Amsden et al. 1992) or GMTEC (Reuss et al. 1995), do not define a priori 
Small scale motion

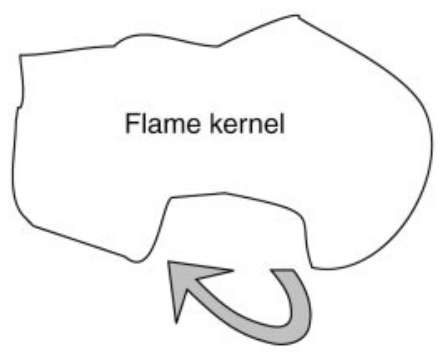

Large scale motion

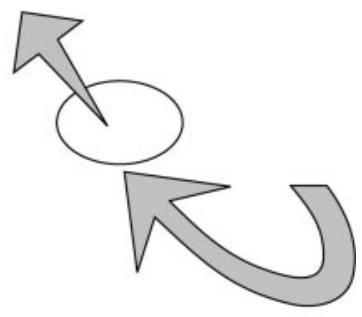

Fig. 1. Comparison of the effect of small scale and large scale motions on the flame kernel

the engine mean velocity. Comparisons between computational model and experimental results have shown some qualitative agreement with mean velocities (Reuss et al. 1995; Delhaye and Cousyn 1996).

\section{2}

\section{Ensemble average}

The flow field decomposition used in Ensemble averaging is defined as

$\mathbf{U}(\theta, k)=\overline{\mathbf{U}_{E}(\theta)}+\mathbf{u}_{E}^{\prime}(\theta, k)$

where $\mathbf{U}(\theta)$ is the measured instantaneous velocity at a crank angle $\left.\theta, \overline{\mathbf{U}_{E}(5694} \mathrm{h}\right)$ is the Ensemble average, and $\mathbf{u}_{E}^{\prime}(\theta, k)$ is the instantaneous turbulence velocity in cycle $k$ at crank angle $\theta$. Note that both $\overline{\mathbf{U}_{E}(\theta)}$ and $\mathbf{u}_{E}^{\prime}(\theta, k)$ are non-stationary with a dependence on $\theta$.

The use of Ensemble averages assumes that the mean repeats each engine cycle. If there are variations in the mean flow field, Ensemble averaging may extract additional turbulence from the signal. Variations in the mean flow field as seen by point measurements can be the result of precessing swirl or tumble flows (Fansler and French 1987). To test the Ensemble averaging technique, an input signal with a time varying mean is used. In the first cycle, the frequency is 1 (Engine Cycle) $)^{-1}$ with an amplitude of 1 . In cycle 2, the frequency is kept at 1 (Engine Cycle) $)^{-1}$, but with an amplitude of 0.75 . No fluctuations are imposed on either cycle, that is, the turbulence is of zero energy. Ensemble averaging calculates the mean and turbulence velocity shown in Fig. 2. It is evident that this technique defines turbulence to exist where there is none.

\section{3}

\section{Cyclic averaging}

Cyclic averaging decomposes the instantaneous flow into the individual cycle mean velocity $\overline{\mathbf{U}_{C}(\theta, k)}$ and the individual cycle turbulence velocities, $\mathbf{u}_{\mathrm{IC}}^{\prime}(\theta, k)$,

$\mathbf{U}(\theta, k)=\overline{\mathbf{U}_{C}(\theta, k)}+\mathbf{u}_{\mathrm{IC}}^{\prime}(\theta, k)$

where $k$ is the cycle number. The Cycle mean is determined by filtering each cycle and separating it into low frequency (mean) and high frequency (turbulence) velocities. This introduces an arbitrariness to the analysis as the division between high and low frequencies is not clear cut. As part of its definition, the

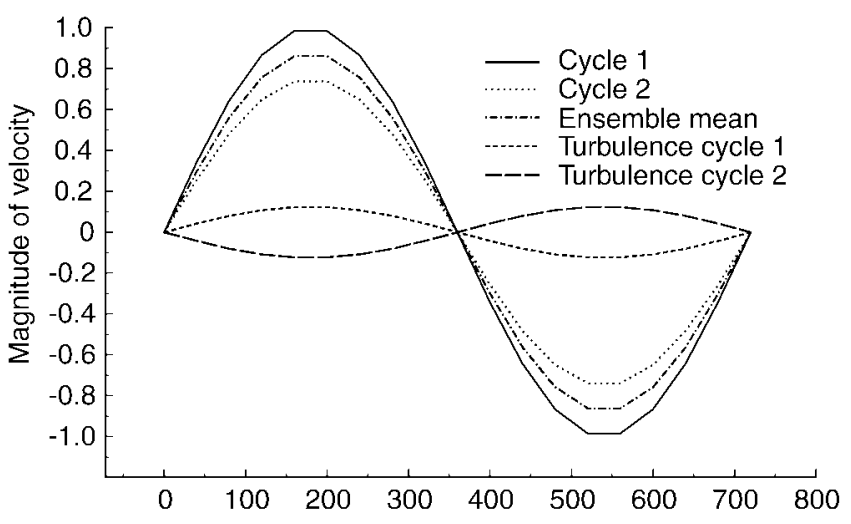

Fig. 2. Ensemble averaged non-stationary flow

ensemble of the low frequency cycles must average to the Ensemble average in Eq. (1).

If the time signal shown in Fig. 2 is analyzed using Cyclic averaging in this manner, the turbulence velocity is correctly defined as zero. The high frequency cut off is easily defined for the example shown in Fig. 2. However, within a measured flow, the definition of the cut-off frequency is not well defined. A systematic definition based on the physical scales of the process is needed, but cannot be made because of variations in the convecting velocity. The variations in convecting velocity from one cycle to the next implies that filtering is done at different length scales for each cycle.

\section{4}

\section{Wavelet averaging}

Wavelet averaging techniques have been introduced recently in engine studies, see (Wiktorsson et al. 1996; Sullivan et al. 1996), and have been successful in extracting coherent structures (quasi-periodic events) within stationary turbulence (Sullivan and Pollard 1996; Higuchi et al. 1994). Unlike Fourier analysis, the basis functions used with wavelet transforms are compact spatially and temporally. This allows decomposition of the measured flowfields with respect to scale.

\subsection{1}

\section{Continuous Wavelet Transform}

The Continuous Wavelet Transform (CWT) is used to perform a spatio-temporal decomposition of a velocity field using a set of predefined basis functions, wavelets. The second derivative of a Gaussian, the Mexican hat, was chosen as the basis for this study,

$g\left(\frac{t}{a}\right)=\left(1-\left(\frac{t}{a}\right)^{2}\right) \exp \left[-\frac{1}{2}\left(\frac{t}{a}\right)^{2}\right]$

where $g$ is the wavelet basis function, $t$ is time and $a$ is a scale parameter. The Mexican hat wavelet was chosen because it an be used to extract maxima or minima of acceleration within a flow (Farge 1992). By increasing or decreasing the value of $a$, the basis function in Eq. 3 is either stretched or contracted. The wavelet must meet the compatibility condition of finite energy (Farge 1992),

$c_{g} \equiv(2 \pi)^{n} \int \frac{|\mathscr{F}(g)|^{2}}{|\omega|^{n}} \mathrm{~d} \omega<\infty$ 
where $n$ is the dimension of the space considered, $\omega$ is a frequency (Engine Cycle) ${ }^{-1}, c_{g}$ is a measure of the total energy in the basis function, $\mathscr{F}$ indicates a Fourier transform, and $n$ the dimension of the space considered. The wavelet transform is a convolution between the wavelet function and the velocity field $u$,

$T_{g}(a, b)=\left(a^{n} c_{g}\right)^{-1 / 2} \iint g^{\star}\left(\frac{t-b}{a}\right) u(b) \mathrm{d} t \mathrm{~d} a$

where $T_{g}$ is the wavelet coefficient and $b$ is a parameter to shift the wavelet. The inverse transform can be defined as (Farge 1992),

$u(t)=c_{g}^{-1} \iint a^{-n-1} T_{g}(a, b) g\left(\frac{t-b}{a}\right) \mathrm{d} a \mathrm{~d} b$

Wavelet transform information can be used to highlight regions of high energy at a particular scale or at a particular time within a time signal. The use of energy methods have been successful identifying energetic motions associated with coherent structures in stationary turbulence (deSouza et al. 1997; Higuchi et al. 1994; Sullivan and Pollard 1996; Sullivan et al. 1994).

\subsection{2}

\section{Discrete Wavelet Transform}

The discrete wavelet transform offers fast localized transformation of time and crank angle information. The signal is decimated with high and low pass filters, into high and low frequency components:

$h(n)=\int_{-\infty}^{\infty} u(t) \phi_{0}^{0}(t) \mathrm{d} t$

$g(n)=\int_{-\infty}^{\infty} u(t) \psi_{0}^{0}(t) \mathrm{d} t$

By operating on the initial signal, it is possible to separate the signal into approximations (low-frequencies, $h(n)$ ) and detail coefficients (high-frequencies, $g(n)$ ). Decimating the approximation coefficients results in further low-pass filtering of the signal. The approximation coefficients will be treated as the mean component of the signal and the detail coefficients as the turbulence component. A Daubechies- 4 wavelet was used with the DWT (Farge 1992). A number of different wavelets were examined; however, there was no significant difference found between the various basis functions.

\section{5}

\section{Use of Wavelet Transforms}

The CWT is used to highlight areas within the flow that show high correlation and to develop energy maps within the flow. For improved calculation speed, the DWT was used to calculate the wavelet based mean; subtracting this mean from the instantaneous velocity field gives the turbulence velocities.

\section{2}

\section{Experimental set-up}

Measurements were made with a Laser Doppler Velocimeter (LDV) within an L-Head research engine (Heywood 1988) modified to provide optical access. A $150 \mathrm{~mm}$ diameter quartz window was fitted to the cylinder head, centered at the original spark plug location. The single top mounted spark plug was replaced by two $10 \mathrm{~mm}$ spark plugs installed in opposing positions on each side of the cylinder head. The LDV was operated in backscatter mode and the flow seeded with $0.2 \mu \mathrm{m}$ diameter $\mathrm{TiO}_{2}$. The engine was motored at wide open throttle at $900 \pm 5$ RPM and data collected using a TSI IFA 750 digital burst correlator controlled with software developed in-house (Ancimer expected 1999). No fired tests were performed for this phase of the study, although the measurement locations near the spark plug were chosen with future fired tests in mind. Details on the research engine are listed in Table 1.

The measurements for this study were performed at three locations (see Fig. 3 for axes definition and Table 2 for position definitions used) and a large number of cycles ( $>1421)$ were sampled for each position.

The $u$ and $v$ velocities are coincident with the $x$ and $y$ coordinate directions. The instantaneous data are pre-filtered using a one crank angle degree coincidence window. Further details on the experimental design are found in (Ancimer expected 1999).

Table 1. Specifications of single cylinder, four stroke engine used in study

\begin{tabular}{ll}
\hline Model & L-Head \\
Bore & $82.8 \mathrm{~mm}$ \\
Stroke & $114.3 \mathrm{~mm}$ \\
Compression ratio & 6.26 \\
Inlet valve open & $15^{\circ}$ ATDC \\
Inlet valve close & $210^{\circ}$ ATDC \\
Exhaust valve open (EVO) & $510^{\circ}$ ATDC \\
Exhaust valve close (EVC) & $15^{\circ}$ ATDC \\
\hline
\end{tabular}

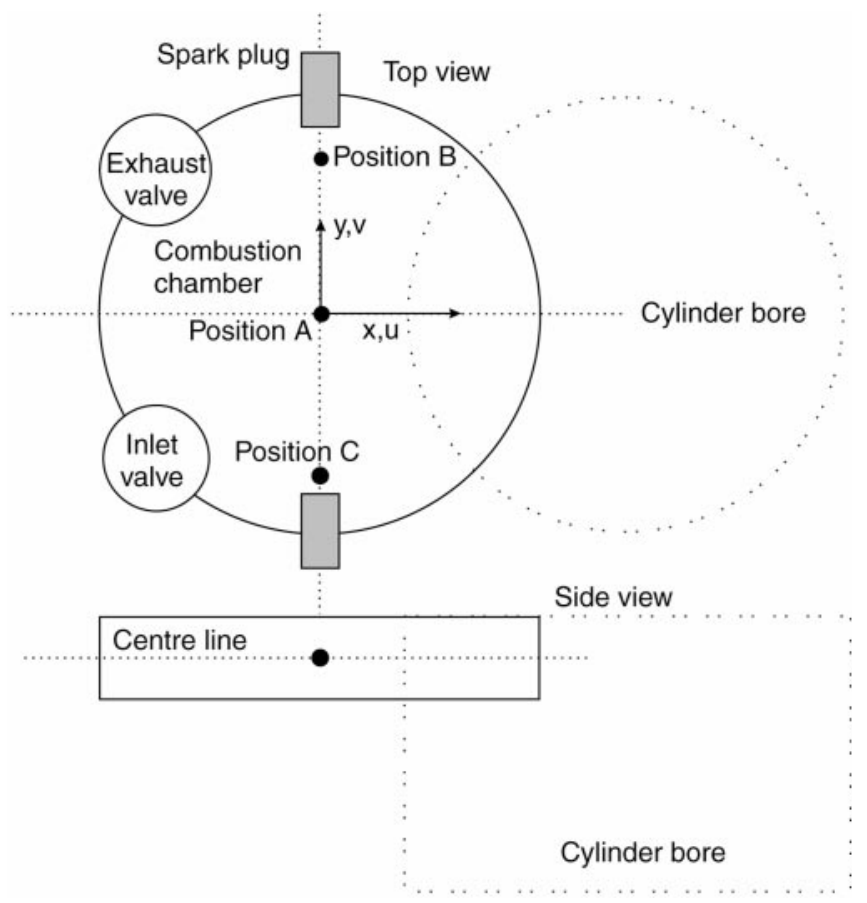

Fig. 3. Combustion chamber geometry showing axes definition 


\section{Experimental results}

The data are presented at three locations within the flowfield. The measurements are compared using (a) Ensemble, (b) Cyclic, and (c) Wavelet based averaging.

\section{1}

\section{Position A}

\subsection{1}

\section{Mean velocities}

At position A, the average mean (large scale) velocities obtained from each method are presented in Figs. 4 and 5 where the individual Cyclic means were filtered at 10(Engine Cycle) ${ }^{-1}$. Both the individual Cycle and the individual DWT means have been ensemble averaged. The results show little quantitative differences for the gross features of the flow. The influence of the inlet $\left(50-200^{\circ}\right)$ and exhaust $\left(500-700^{\circ}\right)$ processes is seen in both the $u$ and $v$-velocities as expected.

The plots in Figs. 6 and 7 show the Ensemble average now compared with the individual Cycle and DWT means for a single cycle (number 40 in the dataset). Comparison of the individual Cycle and DWT means with the Ensemble average velocity indicates that there are significant cycle to cycle variations in the flow field.

The Ensemble average results are significantly different from the Cyclic and DWT averages; however, the Cyclic and DWT results show similar behaviour. Thus, while all techniques will have the same cyclo-stationary property (the ensemble),

Table 2. Measurement positions for this study

\begin{tabular}{ll}
\hline Position & Total number of cycles \\
\hline A $(0,0,10)$ & 1421 \\
B $(0,20,10)$ & 1849 \\
C $(0,-25,10)$ & 1492 \\
\hline
\end{tabular}

non-stationarities affect the individual realizations of engine turbulence.

\subsection{2}

\section{Wavelet analysis}

The total signal was analyzed with CWT using the Mexican hat wavelet. The data for cycle 40 are presented in Figs. 8 and 9. These plots are correlation coefficient energy contour plots, $T_{g}^{2}$. The ordinate is the dilation scale $a$ in Eq. (3). Large values on the ordinate correspond to small scales (large $a$ ) and small values correspond to large scales ( $\operatorname{small} a$ ). The abscissa is the engine cycle CAD. The plots in Figs. 8 and 9 indicate that the jets formed by the inlet and the exhaust processes are captured by the wavelet decomposition. This is expected, as measurements at the midpoint of the combustion chamber should capture both the inlet and exhaust events.

Because they are assumed to be quasi-periodic, it was expected that energetic scales found with the CWT will be in individual cycles and also in the ensemble average of the CWT over all cycles. This is shown in Figs. 10 and 11 which are ensemble averages of the $u$ and $v$-velocities CWT maps of all cycles measured at Position A. The same range of energetic scales is also found in Fig. 8, which is an individual cycle (number 40) measured at Position A. This further suggests that there exists an important component to the flow which, while not completely periodic, does repeat cycle to cycle. Comparing the total $u$ and $v$-velocity CWT decompositions, it is apparent that there exists different scales that are important in both flows. The $u$-velocity has a peak that occurs at approximately scale 20 (corresponding to 7 (Engine Cycle) ${ }^{-1}$ ) and a second

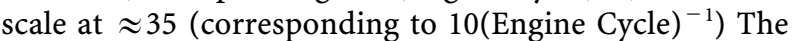
$v$-velocity has peaks at $\approx 15$ (corresponding to 1 (Engine Cycle $)^{-1}$ ) and $\approx 35$ (corresponding to 10 (Engine Cycle) ${ }^{-1}$ ) The $v$-velocity peak only occurs in the signal from $0-200^{\circ}$. There is little correspondence of scales between the $u$ and $v$ velocities. This suggests that the current practice of using uniform filters, or merely using one scale at approximately 80 (Engine Cycle) ${ }^{-1}$, on the velocity field is not appropriate.

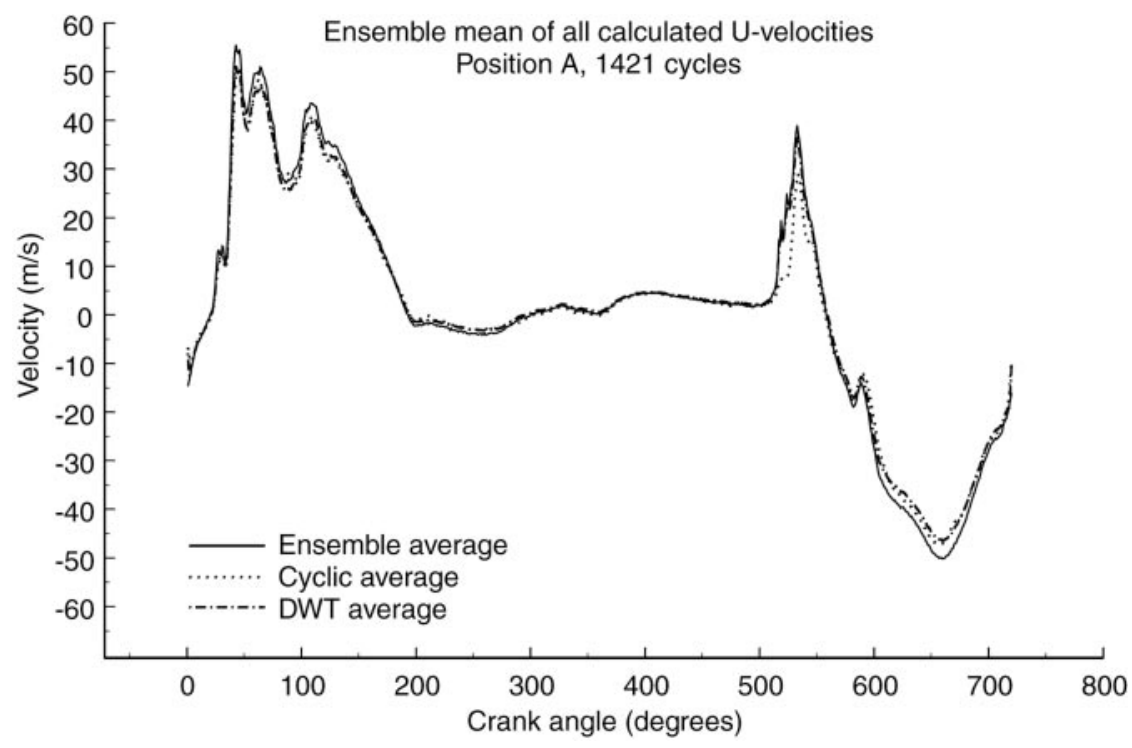

Fig. 4. Ensemble mean $u$-velocities obtained at position A for (a) Ensemble, (b) Cycle and (c) DWT average 

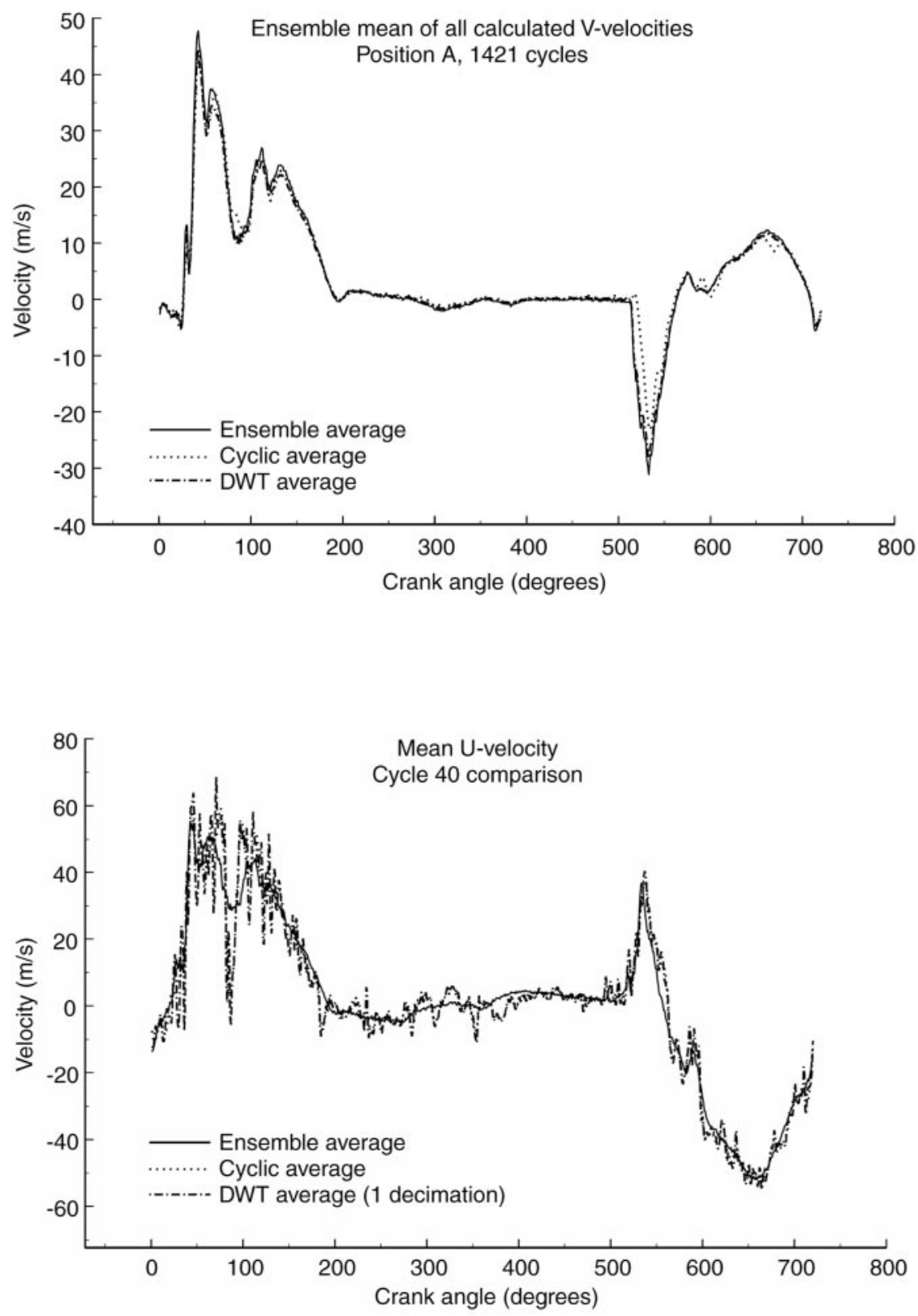

Fig. 5. Ensemble mean $v$-velocities obtained at position A for (a) Ensemble, (b) Cycle and (c) DWT average
Fig. 6. Mean $u$-velocities obtained at position A for cycle number 40 (a) Ensemble, (b) Cycle and (c) DWT average
Examining the flow map in Figs. 10 and 11, it is also noted that there are distinct scales for each stroke of the engine. Filter scales appropriate for each engine cycle stage must be chosen.

\subsection{3}

\section{PSD}

Comparison is also made between the Power Spectral Densities (PSD) obtained from the three averaging techniques. Note that all PSDs were obtained with even crank-angle sampling. Unlike stationary flows, the mean is not limited to a DC contribution. The mean velocity PSD are shown in Figs. 12 and 13. The energy in the Ensemble mean occurs at all measurement frequencies, unlike in the Cyclic average where, because of the cut-off frequency chosen, the drop-off occurs at 80(Engine Cycle) ${ }^{-1}$. The DWT average shows similar behaviour as that of the Cyclic average, with a divergence at 80 (Engine Cycle) $^{-1}$. The Cyclic average PSD has a sharp drop-off, the DWT with 1 level of decomposition average PSD has a slow roll-off and the DWT with 2 levels of decomposition shows ringing. The ringing occurs in the overlap region between the first and second level DWT filters. The different behaviours of the DWT at 1 and 2 levels of decomposition indicates a potential weakness of DWT averaging; there is a subjectivity in defining the appropriate number of decompositions.

The turbulence velocity PSD for the three averaging techniques are shown in Figs. 14 and 15. The high frequency limit (above 80 (Engine Cycle) $^{-1}$ ) to the PSD are the same for all averaging techniques. The Ensemble average has components of the turbulence from the lowest to highest frequencies.

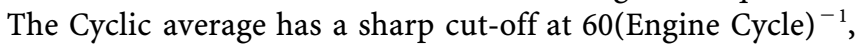




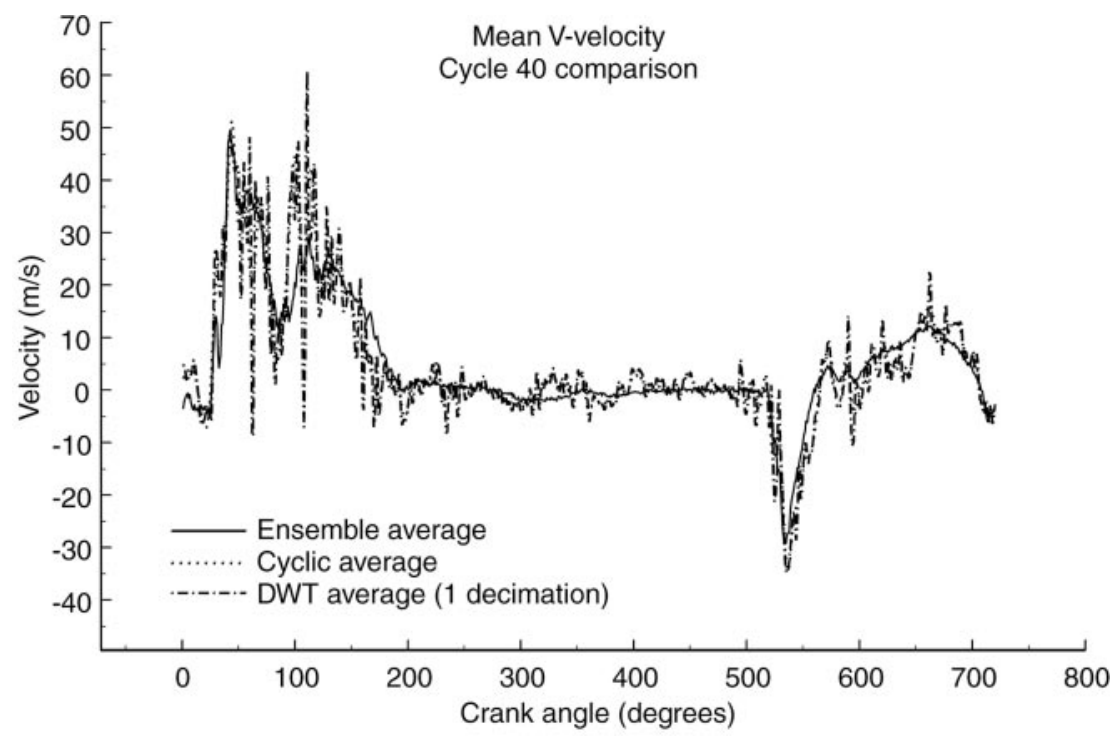

Fig. 7. Mean $v$-velocities obtained at position A for cycle number 40 (a) Ensemble, (b) Cycle and (c) DWT average

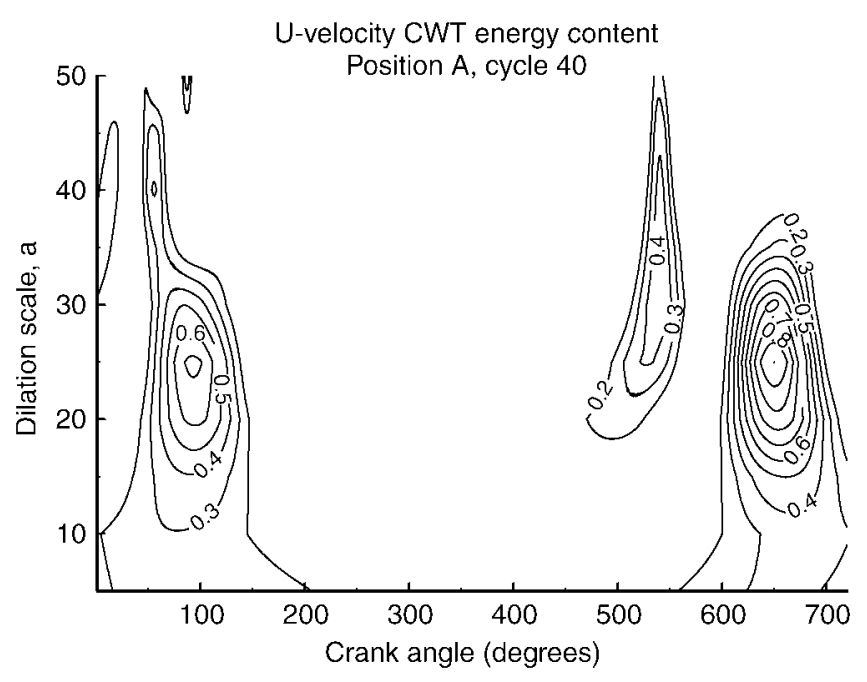

Fig. 8. Square of CWT coefficients for $u$-velocity at cycle 40 , normalized by maximum energy

while the DWT shows a slow build up from approximately 30(Engine Cycle) ${ }^{-1}$. The energy in the turbulence extracted with the DWT average with only one decimation is significantly less than that found with two decimations of the signal. The frequency content is also slightly different; however, both DWT PSDs have similar slopes and behaviour. The turbulence energy (integrated over crank-angle) is a factor of two greater for the turbulent field obtained from Ensemble averaging than that found with either DWT or Cyclic averaging.

\section{2}

\section{Position B}

\subsection{1}

\section{Mean velocities}

Position B is located near the wall on the exhaust valve side of the combustion chamber. The mean velocities are plotted in

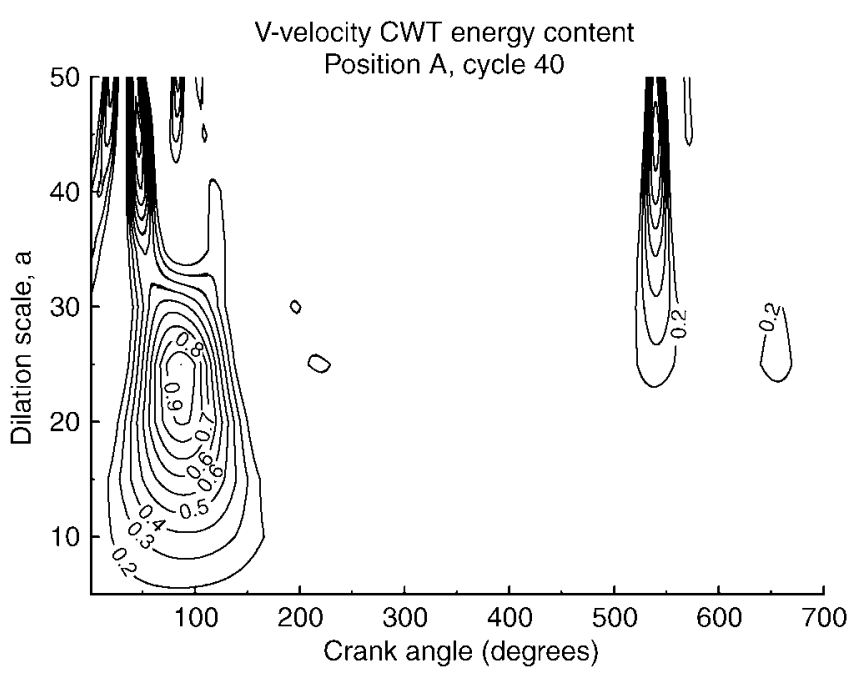

Fig. 9. Square of CWT coefficients for $v$-velocity at cycle 40, normalized by maximum energy

Figs. 16 and 17 . The $u$-velocities are significantly different than those found at Position A which is in the middle of the combustion chamber. The $u$-velocity magnitude is dampened by the proximity of the measurement point to the wall. The $v$-velocities show similar behaviour (an influence from the inlet and exhaust events) as found in the centre of the combustion chamber.

\subsection{2}

\section{Wavelet analysis}

The CWT energy plots are plotted for the $u$ and $v$-velocities, Figs. 18 and 19. The peak scales of the $u$-velocities are only located during the exhaust process of the cycle with the peak scales at similar scale as those found at Position A. The inlet process does not have as significant an influence on the $u$-velocity at Position B. It does not appear with a significant 


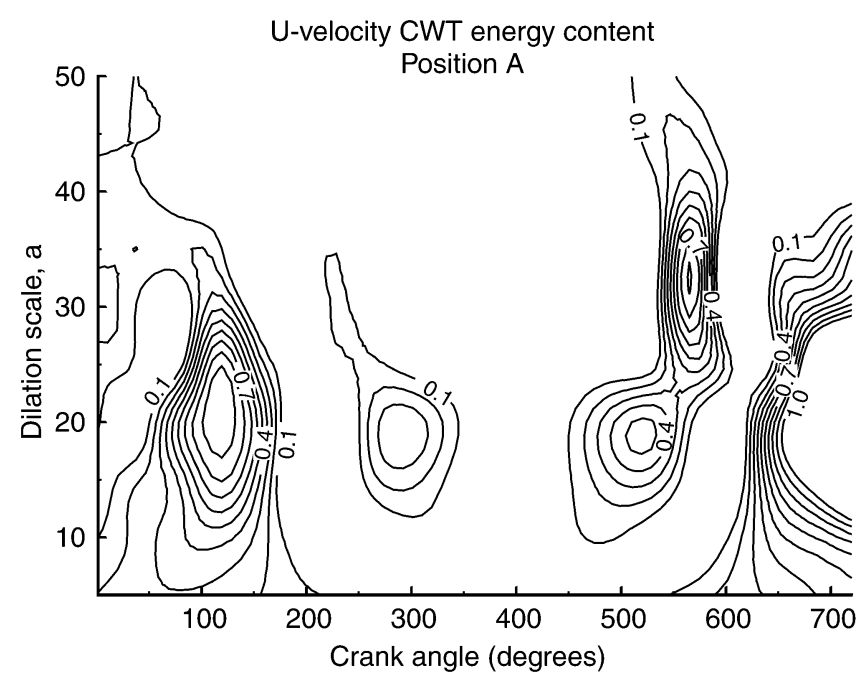

Fig. 10. Square of CWT coefficients for all cycles for the $U$-Velocity, normalized by maximum energy

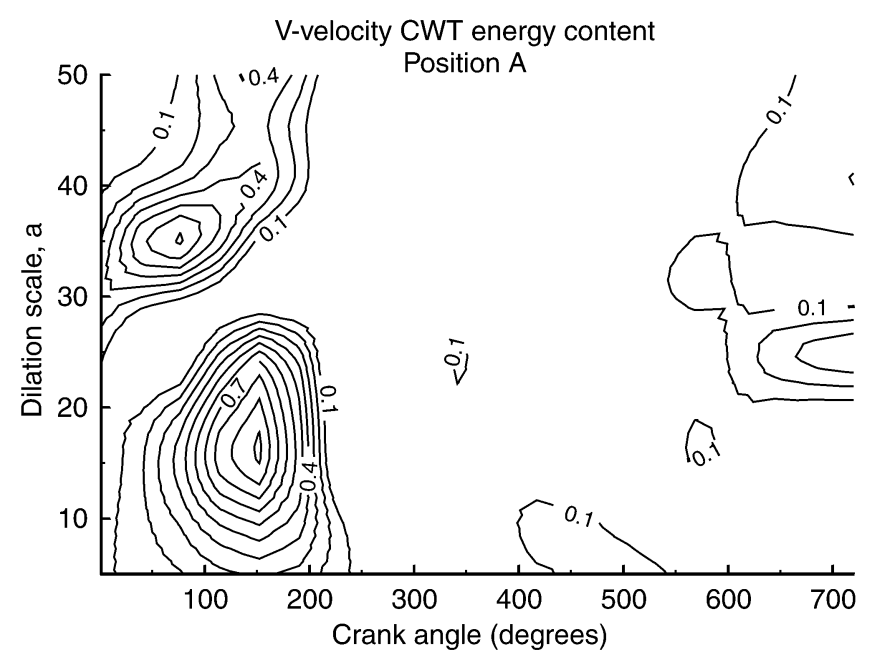

Fig. 11. Square of CWT coefficients for all cycles for the $V$-Velocity, normalized by maximum energy

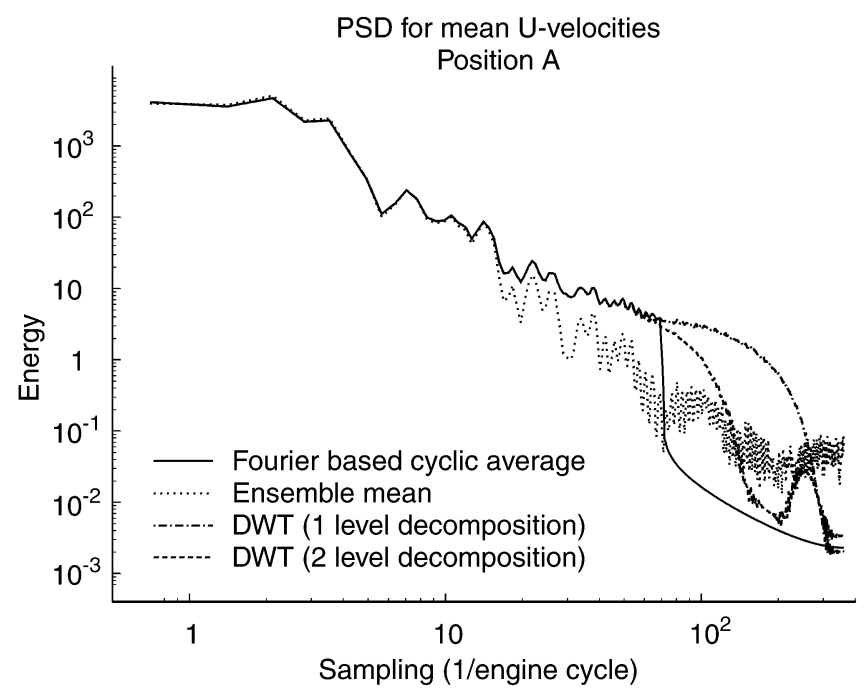

Fig. 12. Mean $U$-velocity PSD for different averaging techniques

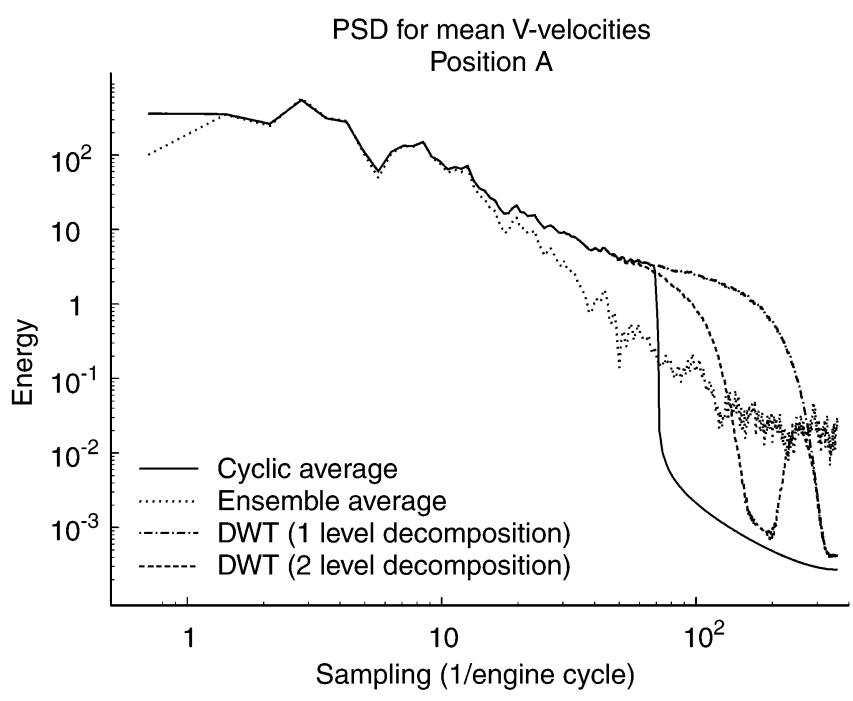

Fig. 13. Mean $V$-velocity PSD for different averaging techniques

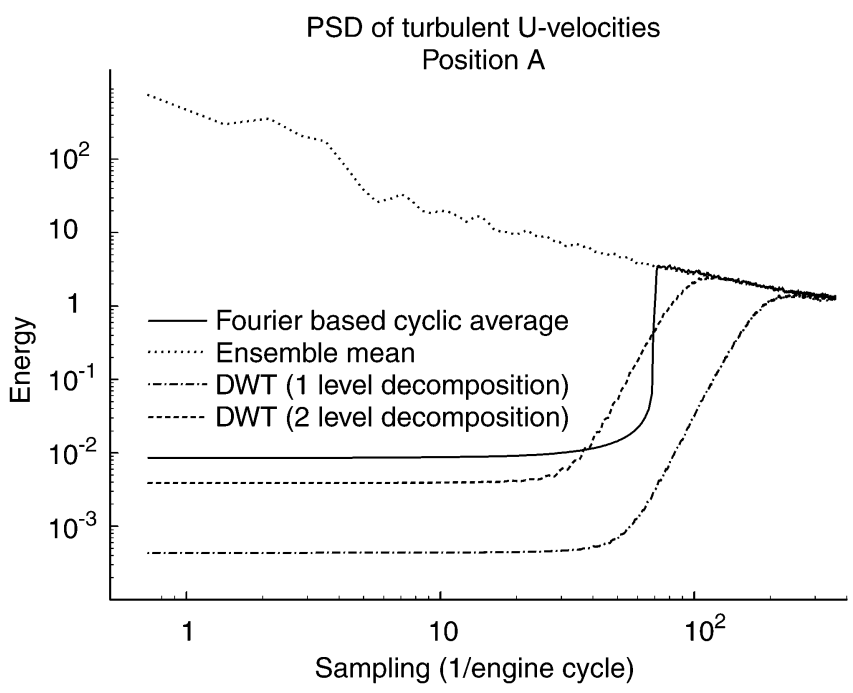

Fig. 14. Turbulence $U$-velocity PSD for different averaging techniques

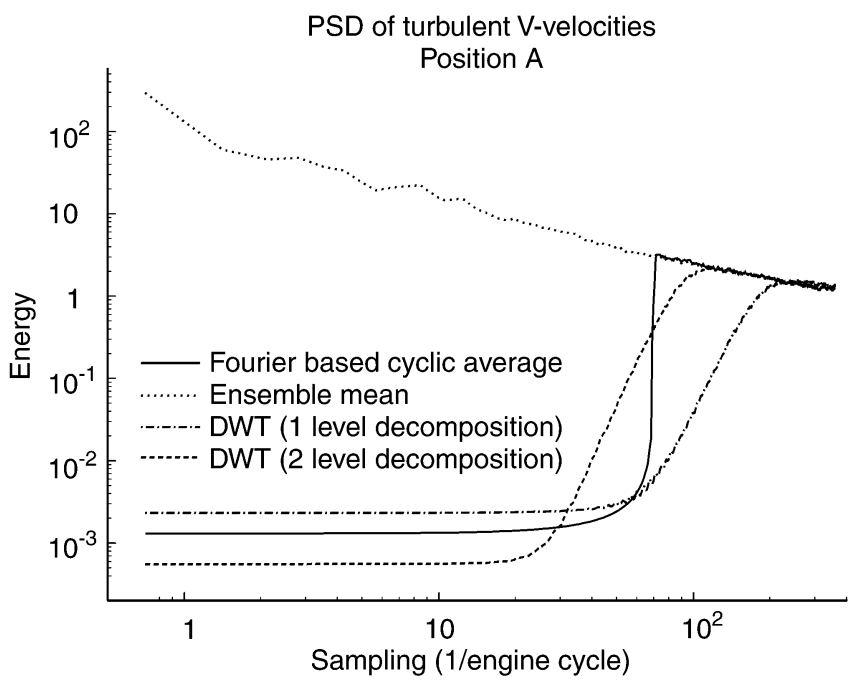

Fig. 15. Turbulence $V$-velocity PSD for different averaging techniques 

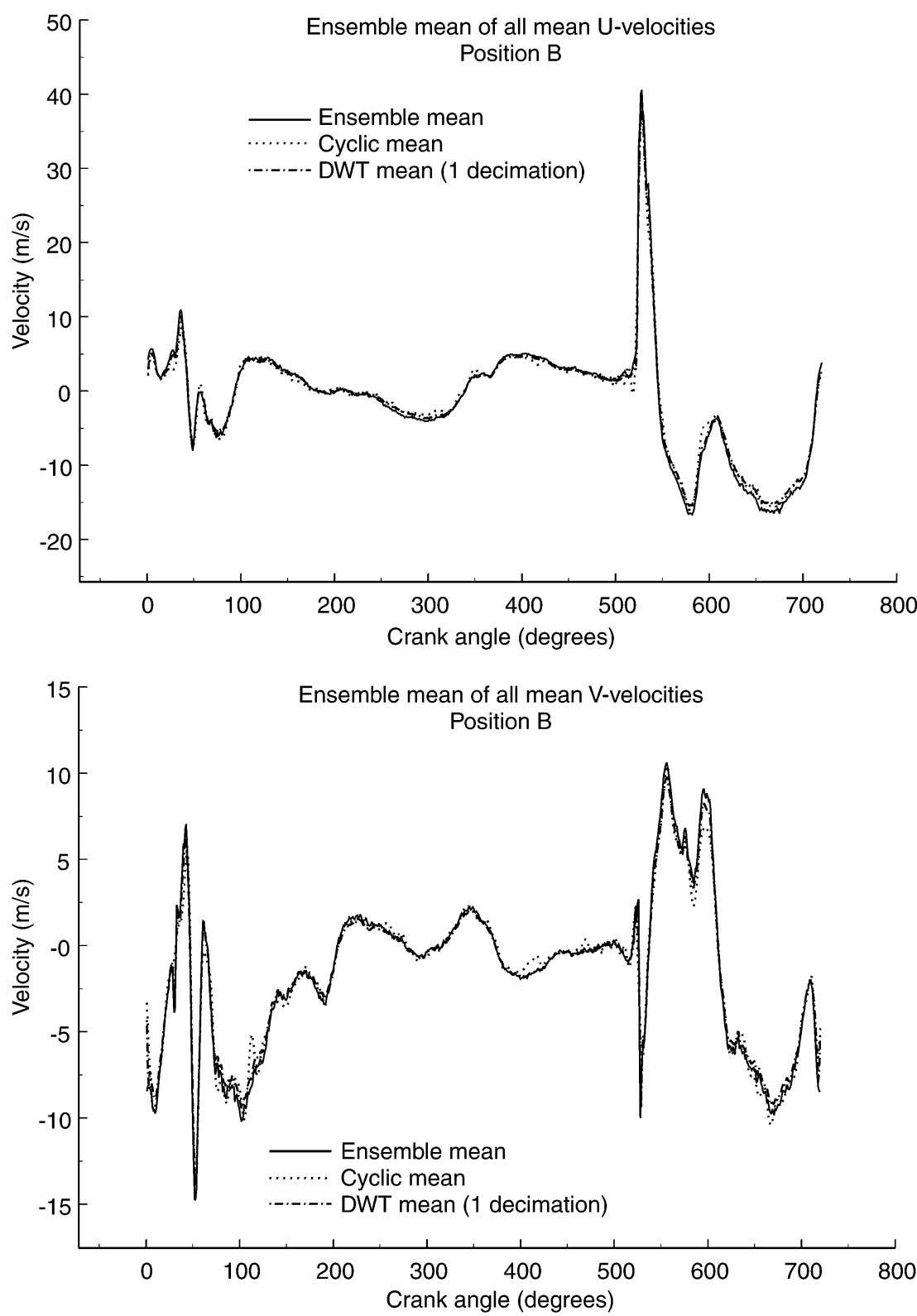

Fig. 16. Ensemble mean $U$-velocities obtained at position B (a) Ensemble, (b) Cycle and (c) DWT average

Fig. 17. Ensemble mean $V$-velocities obtained at position B (a) Ensemble, (b) Cycle and (c) DWT average
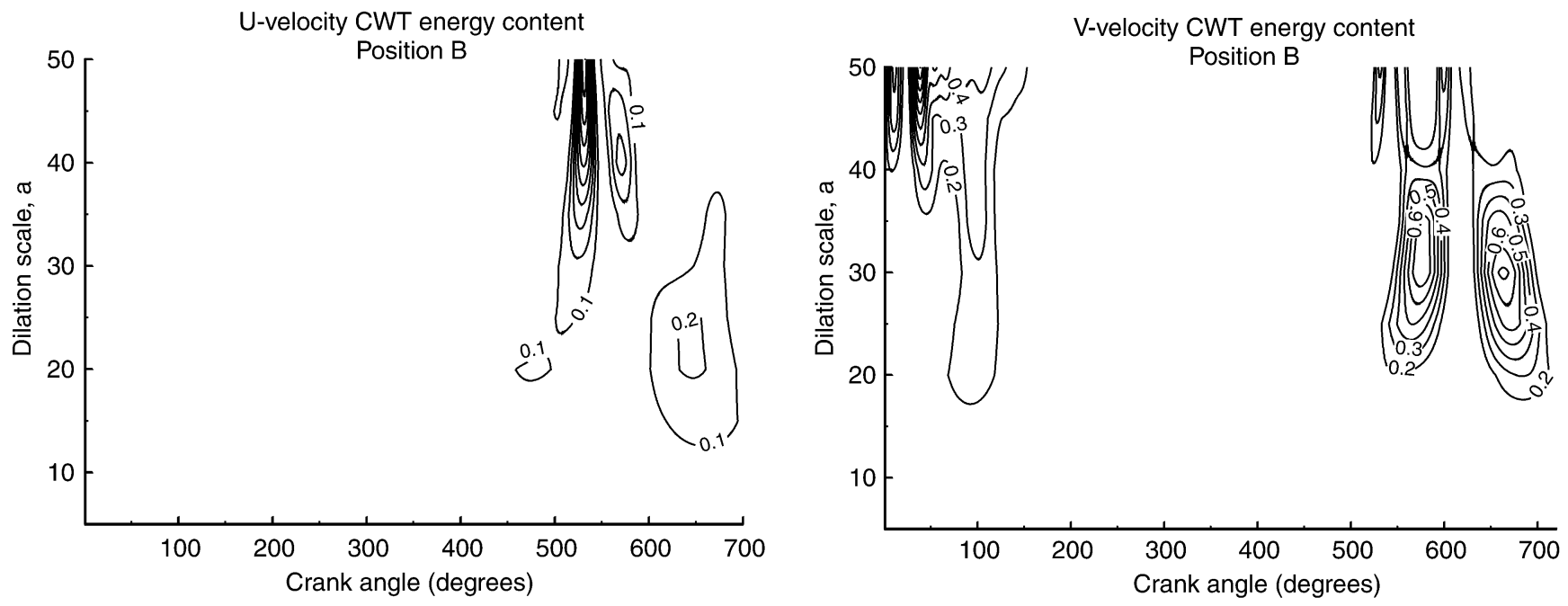

Fig. 18. Square of CWT coefficients for all cycles for the $U$-velocity, normalized by maximum energy

Fig. 19. Square of CWT coefficients for all cycles for the $V$-velocity, normalized by maximum energy 
energy in the CWT energy. The $v$-velocities show the influence of the inlet and exhaust events, with similar scales as those found for the $v$-velocity at Position A. There are also significantly larger scales. Again, the jets formed by the inlet and exhaust processes are captured by the wavelet decomposition and the energetic motions (the mean) are well described by the CWT.

\section{3}

\section{Position C}

\subsection{1}

\section{Mean velocities}

Position $\mathrm{C}$ is located near the wall of the combustion chamber on the inlet valve side opposite Position B. The mean velocities are plotted in Figs. 20 and 21 . The $u$-velocities are significantly
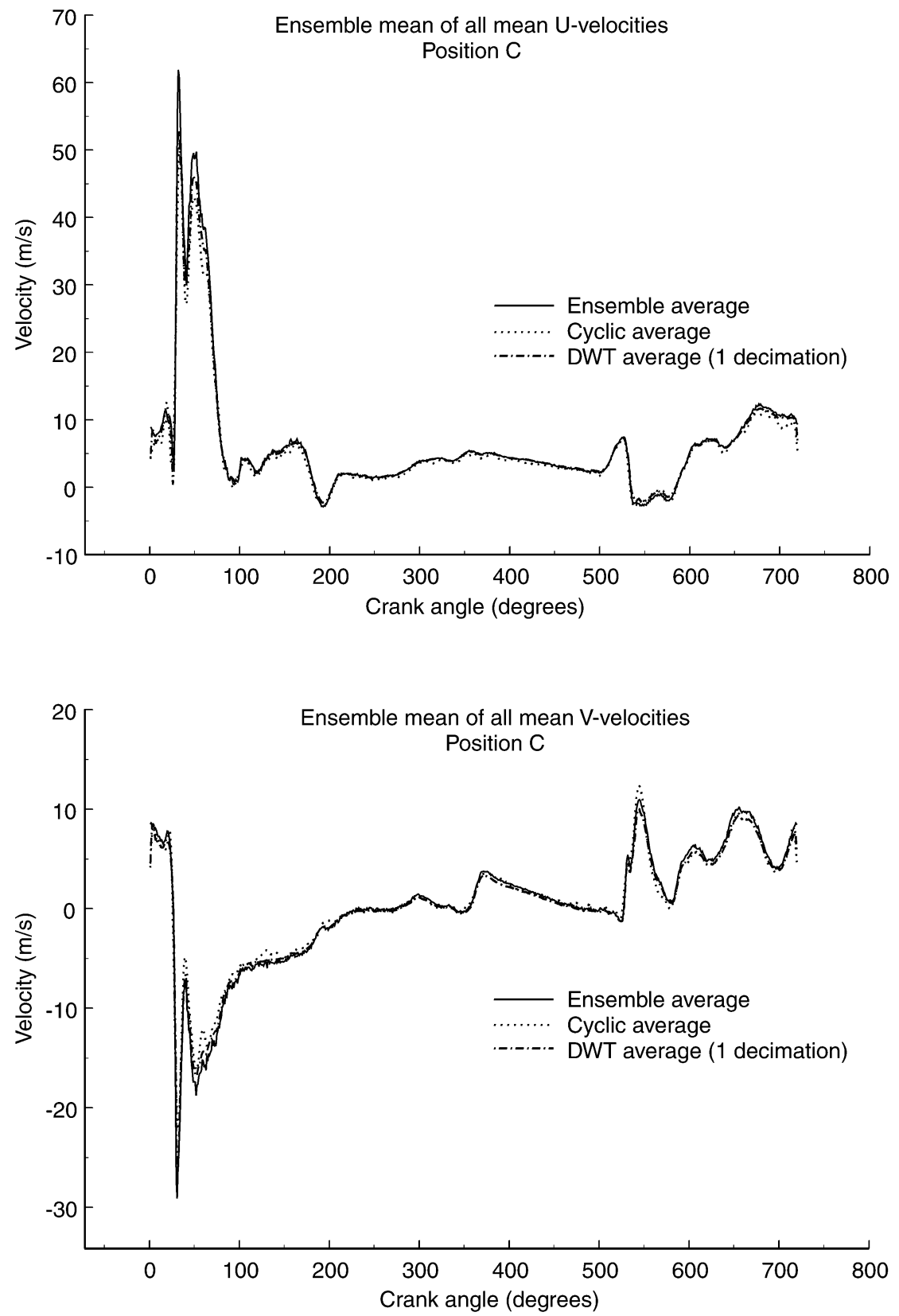

different than those found at Position A. The intake process is evidently of high energy, and no evidence is captured of the exhaust process. The $v$-velocities shows similar behaviour as found in the centre of the combustion chamber.

\subsection{2}

\section{Wavelet analysis}

The CWT energy plots are plotted for the $u$ and $v$-velocities, Figs. 22 and 23. The peak scales of the $u$-velocities are only located in the first $100^{\circ}$ of the cycle. The mean $u$-velocity is influenced by the intake jet, not the exhaust jet. The important intake process energetic scales are similar to those found at Position A; however, scales important at Position A (e.g., 25) do not appear at this position. The peak scales are at the same CAD location as those found at Position A. The $v$-velocities show similar behaviour as found at Position $\mathrm{A}$ and $\mathrm{B}$.
Fig. 20. Ensemble mean $U$-velocities obtained at position C (a) Ensemble, (b) Cycle and (c) DWT average

Fig. 21. Ensemble mean $V$-velocities obtained at position C (a) Ensemble, (b) Cycle and (c) DWT average 


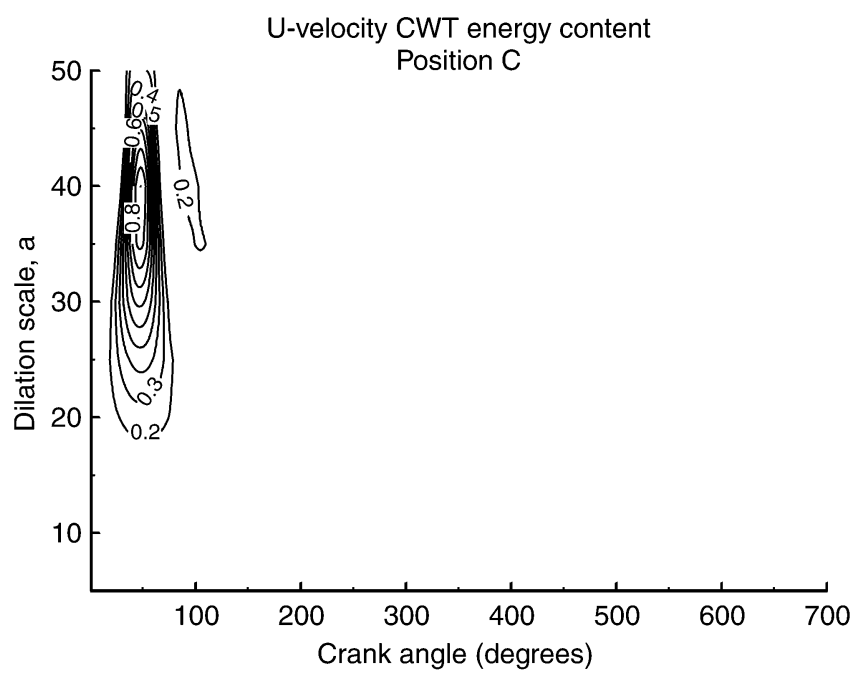

Fig. 22. Square of CWT coefficients for all cycles for the $U$-velocity, normalized by maximum energy

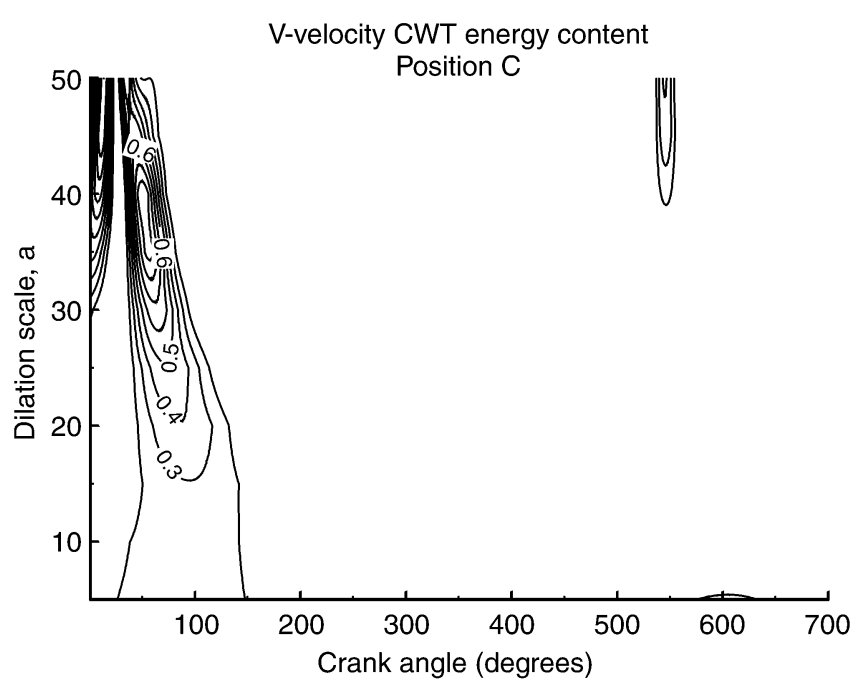

Fig. 23. Square of CWT coefficients for all cycles for the $V$-velocity, normalized by maximum energy

4

\section{Conclusions}

The wavelet analysis allows for the determination of the mean and turbulence velocity fields without any assumptions with respect to the periodicity of the flow. The use of this technique allows better insight into the mechanics of flow in SI engines. The clearer separation of turbulent and mean motions is the advantage of combining the DWT and CWT techniques.

The important results of this work are (a) an indication that there exists a wide range of scales that are important to the mean motion and have been identified with CWT, (b) the variety of scales found with the CWT show that uniform cut-off frequencies as used with Cyclic averages are not appropriate, (c) a new DWT based average velocity, (d) that DWT averages suffers from the same subjectivity in implementation as Cyclic averages and (e) Ensemble averages may be inappropriate within SI engine flows.

\section{References}

Amsden A; Butler T; O'Rourke PJ (1992) The KIVA-II computer program for transient multidimensional chemically reactive flows with sprays. Technical Paper Series No. 920418. SAE

Ancimer R (1999) The influence of in-cylinder flows in the combustion process in production spark ignition engine. Unpublished doctorul dissertation, Mechanical and Industrial Engineering, University of Toronto

Catania AE; Mittica A (1989) Extraction techniques and analysis of turbulence quantities from in-cylinder velocity data. Trans ASME: J Eng Gas Turbines Power 111: 466-478

Delhaye B; Cousyn B (1996) Computation of flow and combustion in spark ignition engine and comparison with experiment. Technical Paper Series No. 961960. SAE. (1996 SAE International Fall Fuel and Lubricants Meeting)

deSouza F; Delville J; Lewalle J; Bonnet JP (1997) On the large scale organization of a turbulent boundary layer disturbed by a circular cylinder. In: Eleventh symp. on turbulent shear flows (pp. 33-25-33-30). Grenoble, France. (Submitted for review by J Exper Thermal Fluid Sci)

Fansler, T; French D (1987) Swirl, squish and turbulence in stratified charge engines: LDA measurements and implications for combustion. Tech. Rep. No. 870371. SAE

Farge M (1992) Wavelet transforms and their applications to turbulence. In: Lumley J; Van Dyke M; Reed H (eds) Annual review of fluid mechanics. Annual Reviews, Inc, pp. 395-457

Hall M; Bracco F (1987) A study of velocities and turbulence intensities measured in firing and motored engines. Tech. Rep. No. 870453. SAE

Heywood JB (1988) Internal combustion engine fundamentals. McGraw-Hill. New York: (McGraw-Hill series in mechanical engineering)

Higuchi H; Lewalle J; Crane P (1994) On the structure of a twodimensional wake behind a pair of flat plates. Phys Fluids 6: 297-305

Reuss D; Kuo T-W; Khalighi B; Haworth D; Rosalik M (1995) Particle image velocimetry measurements in a high-swirl engine used for evaluation of computational fluid dynamics calculators. Technical Paper Series No. 952381. SAE

Söderberg F; Johansson B (1998) Wavelet analysis of in-cylinder LDV measurements and correlation against heat-relay. Technical Paper Series No. 980483. SAE. (1998 SAE International Congress and Exposition)

Sullivan P; Ancimer R; Dziedzic M; Wallace J (1996) Analysis of single-point nonstationary data with application to SI engines. Proc CSME forum on Thermal and Fluids Engineering, May 7-9, 1996, McMaster University, Hamilton, Ontario

Sullivan P; Day M; Pollard A (1994) Enhanced VITA techniques for turbulent structure identification. Exp Fluids 18: 10-16

Sullivan P; Pollard A (1996) Coherent structure identification from the analysis of hotwire data. Measurement Sci Technol 1498-1516

Wiktorsson M; Lindoff B; Johansson B; Söderberg F (1996) Wavelet analysis of in-cylinder LDV measurements. Technical Paper Series No. 961921. SAE. (1996 SAE International Fall Fuel and Lubricants Meeting) 